\title{
Influence of the shape and surface oxidation in the magnetization reversal of thin iron nanowires grown by focused electron beam induced deposition
}

\author{
Luis A. Rodríguez $¥ 1,2,3$, Lorenz Deen ${ }^{\ddagger 1,4}$, Rosa Córdoba ${ }^{1,4}$, César Magén $1,2,5$, \\ Etienne Snoeck ${ }^{3}$, Bert Koopmans ${ }^{4}$ and José M. De Teresa ${ }^{* 1,2,6}$
}

\section{Full Research Paper}

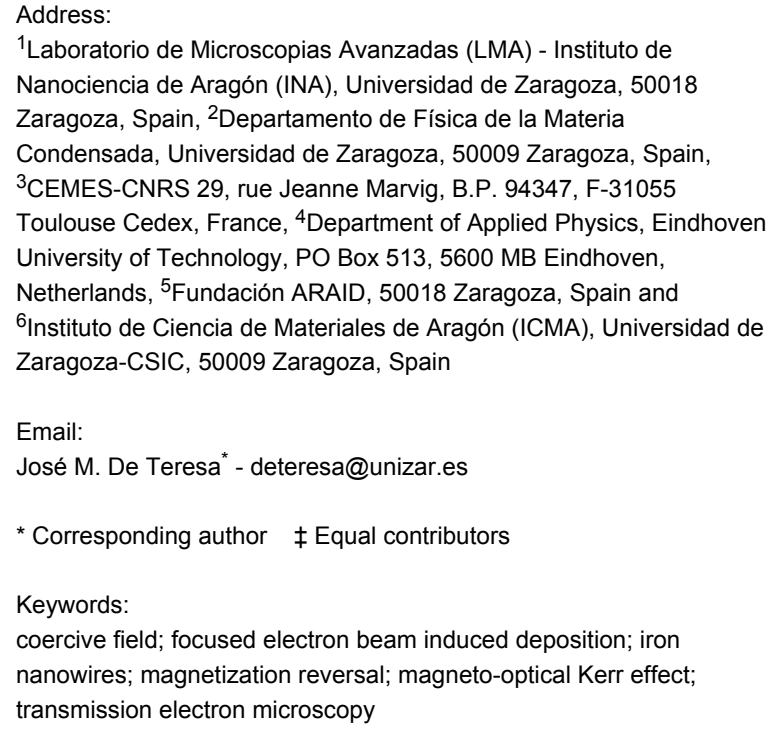

${ }^{1}$ Laboratorio de Microscopias Avanzadas (LMA) - Instituto de Nanociencia de Aragón (INA), Universidad de Zaragoza, 50018 Zaragoza, Spain, ${ }^{2}$ Departamento de Física de la Materia Condensada, Universidad de Zaragoza, 50009 Zaragoza, Spain, ${ }^{3}$ CEMES-CNRS 29, rue Jeanne Marvig, B.P. 94347, F-31055 Toulouse Cedex, France, ${ }^{4}$ Department of Applied Physics, Eindhoven University of Technology, PO Box 513, 5600 MB Eindhoven, Netherlands, ${ }^{5}$ Fundación ARAID, 50018 Zaragoza, Spain and ${ }^{6}$ Instituto de Ciencia de Materiales de Aragón (ICMA), Universidad de Zaragoza-CSIC, 50009 Zaragoza, Spain

\section{Email:}

José M. De Teresa* - deteresa@unizar.es

* Corresponding author $\ddagger$ Equal contributors

\section{Keywords:}

coercive field; focused electron beam induced deposition; iron nanowires; magnetization reversal; magneto-optical Kerr effect; transmission electron microscopy

\begin{abstract}
Beilstein J. Nanotechnol. 2015, 6, 1319-1331. doi:10.3762/bjnano.6.136
\end{abstract}

Received: 06 February 2015

Accepted: 18 May 2015

Published: 15 June 2015

This article is part of the Thematic Series "Focused electron beam induced processing".

Guest Editor: M. Huth

(C) 2015 Rodríguez et al; licensee Beilstein-Institut. License and terms: see end of document.

\begin{abstract}
Iron nanostructures grown by focused electron beam induced deposition (FEBID) are promising for applications in magnetic sensing, storage and logic. Such applications require a precise design and determination of the coercive field $\left(H_{\mathrm{C}}\right)$, which depends on the shape of the nanostructure. In the present work, we have used the $\mathrm{Fe}_{2}(\mathrm{CO})_{9}$ precursor to grow iron nanowires by FEBID in the thickness range from 10 to $45 \mathrm{~nm}$ and width range from 50 to $500 \mathrm{~nm}$. These nanowires exhibit an Fe content between 80 and $85 \%$, thus giving a high ferromagnetic signal. Magneto-optical Kerr characterization indicates that $H_{\mathrm{C}}$ decreases for increasing thickness and width, providing a route to control the magnetization reversal field through the modification of the nanowire dimensions. Transmission electron microscopy experiments indicate that these wires have a bell-type shape with a surface oxide layer of about $5 \mathrm{~nm}$. Such features are decisive in the actual value of $H_{\mathrm{C}}$ as micromagnetic simulations demonstrate. These results will help to make appropriate designs of magnetic nanowires grown by FEBID.
\end{abstract}




\section{Introduction}

The fabrication of magnetic nanostructures in a single lithographic step by focused electron beam induced deposition (FEBID) is currently an exciting research topic [1,2]. In this technique, a scanning electron microscope (SEM) dissociates the precursor molecules delivered into the area of interest by a gas-injection system, producing a deposit [3-6]. The use of precursor molecules containing cobalt [7-10] or iron [11-14] allows for the growth of magnetic nanostructures with tailored dimensions. Some of the most recent advances in this topic are: the achievement of resolution in single magnetic structures below $30 \mathrm{~nm}$ [15-17], the fabrication of nanomagnets for logic [18], the production of highly-dense isolated magnetic structures [19], the growth of three-dimensional nanowires [20,21] and the fabrication of nanospheres on scanning probe tips $[22,23]$.

One of the crucial parameters to be controlled in such magnetic nanostructures grown by FEBID is the coercive field, $H_{\mathrm{C}}$, which corresponds to the magnetic field producing the magnetization reversal. Most of magnetic devices work by producing a voltage output when the magnetization reversal occurs. In the case of cobalt deposits, it was previously found that the coercive field is governed by shape anisotropy [24] due to the polycrystalline microstructure [25], and is thus a function of the deposit dimensions [26]. However, more detailed studies subsequently emphasized the role played by the halo and the effective magnetic shape in the coercive field of cobalt nanowires [27-29]. In brief, the halo structure around the main deposit (caused by proximity effects in FEBID [30]) is an easy place for nucleation of domain walls (DWs) [31] starting the magnetization reversal of the nanowire, which gives rise to low coercive fields. This can be a source of troubles if one naively aims to control the coercive field of magnetic nanostructures by means of the shape of the main deposit without taking into account the effect of the halo.

Regarding the case of iron nanostructures grown by FEBID, the work by Gavagnin et al. has highlighted that the coercive field could be controllable by means of the deposit thickness [18]. These authors found that nanomagnets with thicknesses of 25 and $35 \mathrm{~nm}$ (but equal length and width) produced different coercive fields [18]. However, a detailed explanation for such phenomenology was not provided. The same group later found that, similar to the case of cobalt nanowires grown by FEBID, a magnetic halo in iron nanowires is an easy nucleation center for domain walls, decreasing the observed coercive field [31].

At this point, further research towards a deeper understanding of the processes that determine the coercive field in magnetic nanostructures grown by FEBID is required. A true control over coercivity is the only way to design and fabricate appropriate magnetic devices based on FEBID materials. In the present work, we focus our attention on iron nanowires with fixed length $(4 \mu \mathrm{m})$ and varying width $(50-500 \mathrm{~nm})$ and nominal thickness $\left(t_{\text {Nom }}\right)$ between 10 and $45 \mathrm{~nm}$. We have chosen this geometry given our previous experience with cobalt nanowires grown by FEBID [24,27]. In these experiments mono-domain magnetic structures in remanence, as required in most applications, were obtained for nanowire widths around $400 \mathrm{~nm}$ or smaller and length/width aspect ratios of the order of 10. By performing systematic studies of the coercive field as a function of dimensions and carrying out micromagnetic simulations, we are able to conclude that the specific shape of the nanowire as well as the surface oxidation are key to explain the observed behavior. These will determine the effective magnetic shape of the nanowires, which will control the coercivity. This will be still more relevant as the dimensions of the nanowires become smaller, as it is desired in some applications where high integration of magnetic nanostructures is needed.

\section{Results and Discussion Growth of the nanowires}

The iron nanowires have been grown on B-doped Si substrates inside an FEI Helios 600 apparatus, using $\mathrm{Fe}_{2}(\mathrm{CO})_{9}$ as precursor and the scanning electron microscope (SEM) to produce magnetic deposits in a single step, as sketched in Figure 1a. The precursor is delivered to the area of interest through a single gas-injection-system (GIS) with inner diameter of $160 \mu \mathrm{m}$, whose tip is located approximately $150 \mu \mathrm{m}$ above ( $z$ direction) and $50 \mu \mathrm{m}$ off ( $x$ direction) the central irradiation point of the electron beam. The base pressure inside the chamber (before the flow of the precursor gas) is about $1 \times 10^{-6}$ mbar. The precursor flux can be controlled through a leak valve in the GIS and had to be optimized in order to obtain deposits exhibiting ferromagnetic properties with suitable shape anisotropy. In these experiments, the nominal turbopump speed is $260 \mathrm{~L} / \mathrm{s}$ for nitrogen gas. When the leak valve is opened, the chamber pressure increases. The chamber pressure is monitored through a Penning vacuum gauge, which serves to perform systematic quantitative studies (we cannot provide direct values of precursor flux on the substrate because the pumping efficiency of the vacuum pump for $\mathrm{Fe}_{2}(\mathrm{CO})_{9}$ molecules in our apparatus is unknown). The beam current and voltage used for the growth were, respectively, $1.4 \mathrm{nA}$ and $3 \mathrm{kV}$. The first experiments under relatively high precursor flux (process pressure approx. $6 \times 10^{-6} \mathrm{mbar}$ ) indicated that the microstructure of the deposits consisted of grains with a typical size of about $100 \mathrm{~nm}$, as can be observed in Figure S1 of Supporting Information File 1. In those wires, there was no significant modification of the coercive field as a function of 



(c)



Figure 1: (a) A sketch of the FEBID process. (b) A SEM image of the nanowire with targeted width of $250 \mathrm{~nm}$ and nominal thickness of $15 \mathrm{~nm}$. (c) Sketch of the two batches of nanowires: Batch 1 has got $25 \mathrm{~nm}$ nominal thickness and varying width whereas batch 2 has got $250 \mathrm{~nm}$ width and varying thickness.

the deposit dimensions, indicating that the magnetization reversal was governed by the individual grains, somehow magnetically decoupled one from each other. However, when the process pressure was decreased to the range from $3 \times 10^{-6}$ to $4 \times 10^{-6}$ mbar, the deposits did not show the granular structure and the magnetization reversal was found to be dominated by shape anisotropy (see next section). In situ compositional analysis by energy dispersive X-ray spectroscopy (EDS) indicated that the $\mathrm{Fe}$ content in these optimized deposits were always in the range of $80-85 \%$, the rest being $\mathrm{C}$ and $\mathrm{O}$. These values are similar to those found by Gavagnin et al. with the precursor $\mathrm{Fe}(\mathrm{CO})_{5}$ [18]. In Figure S2 of Supporting Information File 1, we show one of the typical EDS spectra of these deposits.

In the following, we will describe the results obtained in two batches of samples corresponding to optimized nanowires. In both batches, the length is fixed to $4.5 \mu \mathrm{m}$ and the ends are triangular (see Figure 1c), as frequently used in this type of nanowires to avoid easy nucleation of domain walls at the end of the nanowire and thus the appearance of low coercive fields. In the first batch, $t_{\mathrm{Nom}}$ is fixed to $25 \mathrm{~nm}$ and the width is varied from 50 to $500 \mathrm{~nm}$ (4 samples). In the second batch, the width is fixed to $250 \mathrm{~nm}$ and $t_{\mathrm{Nom}}$ is varied from 10 to $45 \mathrm{~nm}$ (8 samples). This will allow for a systematic study of the effect of varying width and thickness in these nanowires.

\section{MOKE experiments: coercive field}

Magneto-optical Kerr effect (MOKE) experiments have been carried out using the Nano-MOKE-3 apparatus by Durham Magnetooptics. This device uses a $2 \mu \mathrm{m}$ diameter infrared laser beam that scans the area of interest in raster mode to detect MOKE contrasts via the variation of the signal amplitude or the change in the rotation angle of the light. The positioning of the beam permits the localization of the nanostructure to be measured and to position the beam in a targeted place. For measurements as a function of the magnetic field, the laser beam is fixed on the centre of the nanowire and quadrupole coils are used to apply a magnetic field in the plane of the sample, which allows for tracing the MOKE changes versus the magnetic field. The MOKE signal is proportional to the total magnetization, allowing one to study magnetization reversal processes and precise determination of the coercive field of the nanowires. In our experiments, we have measured the longitudinal MOKE signal with the magnetic field being applied parallel to the nanowire length (easy axis) [32]. 
As an example of the type of magnetization loops measured, we show in Figure 2a and Figure $2 b$ the results for two nanowires from the second batch with $t_{\text {Nom }}$ of 10 and $35 \mathrm{~nm}$. The signalto-noise ratio is ten, certainly enough to determine the coercive field, which is given by the measured MOKE field at the midpoint between the two saturation values at high positive and high negative fields. The observed noise, about $10 \%$ of the signal, is small given that the nanowire width is only $1 / 8$ of the laser diameter (thus having significant signal originated from the non-magnetic substrate). The values of $H_{\mathrm{C}}$ for both batches are represented in Figure $2 \mathrm{c}$ and Figure 2d. All results shown in Figure 2 correspond to the average of more than 100 hysteresis loops. Given the sharp switching transitions observed, a high level of reproducibility can be inferred.

The observed decrease of $H_{\mathrm{C}}$ with the width was also observed in polycrystalline cobalt nanowires grown by FEBID with widths above $250 \mathrm{~nm}[26,27]$ and previously in permalloy [33] and cobalt [34-36] nanowires patterned by electron beam lithography. Such dependence was explained by a model in which a small volume in the wire reverses magnetization coherently, propagating across the entire wire. In such a model, the coer- cive field is proportional to the ratio thickness $(t) /$ width $(w)$ due to demagnetizing effects:

$$
H_{\mathrm{C}}=H_{\infty}+a(t / w)
$$

where $H_{\infty}$ is the coercive field for a wire with infinite width (thin film) and $a$ is constant parameter that depends of the finite-length shape anisotropy factor and saturation magnetization [37]. Applying this model to the Fe wires of the batch 1, we obtain that $H_{\infty}$ is $7 \mathrm{Oe}$, indicating that the material is soft magnetic, as expected for $\mathrm{Fe}$.

We note, however, that the model is not able to explain the thickness dependence of $H_{C}$ in the $250 \mathrm{~nm}$ wide Fe nanowires of the batch 2 . In fact, we observe that $H_{\mathrm{C}}$ decreases with increasing thickness, contrary to the model. Similar behavior has also been observed theoretically in permalloy nanowires [38] and experimentally in permalloy nanowires arrays [39] and in cobalt nanowires grown by FEBID [40]. In all cases, the occurrence of the failure of the model is caused by a transition in the type of magnetization reversal process, which led us to
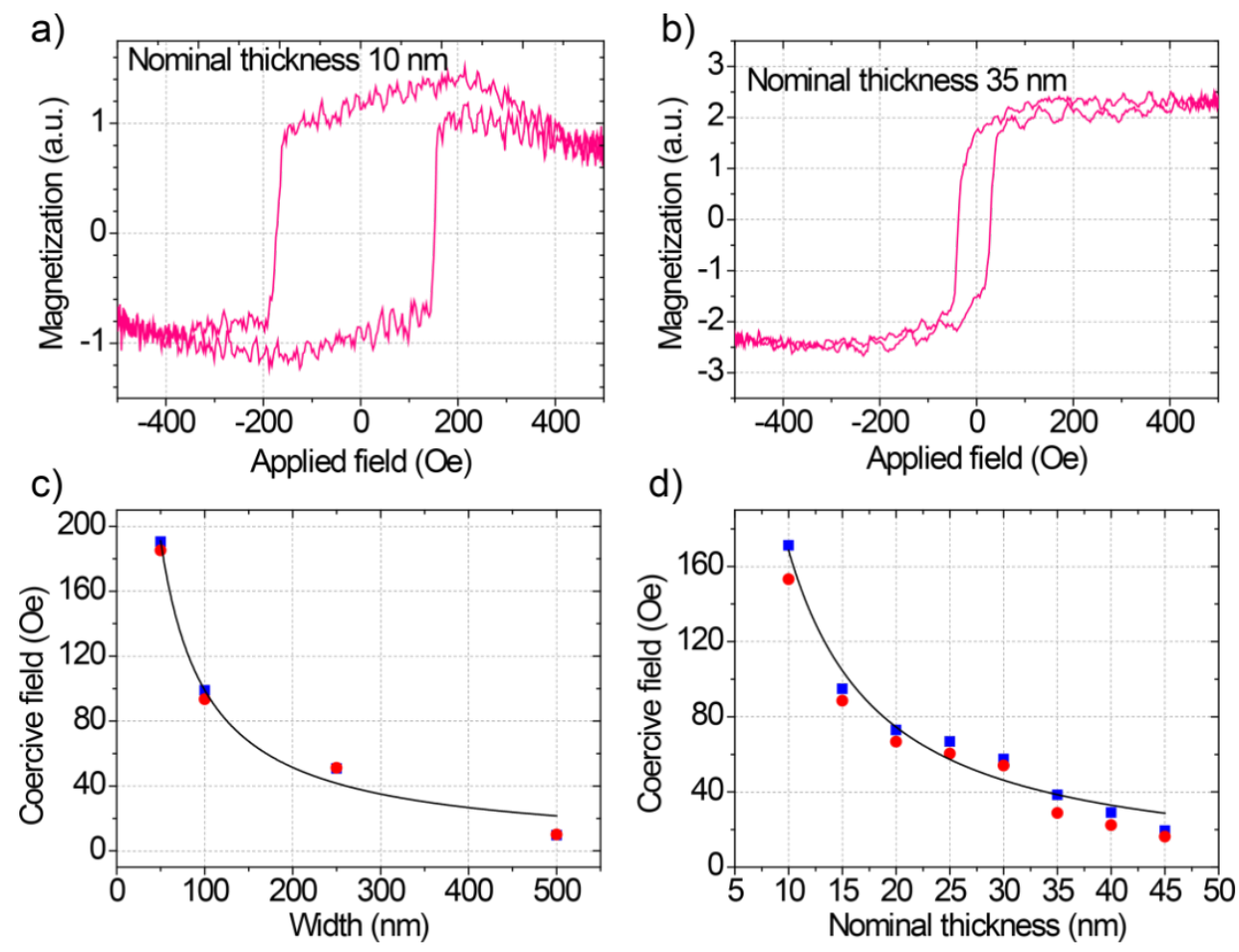

Figure 2: MOKE results. (a) Average magnetic hysteresis loop of the sample with width/nominal thickness of $250 \mathrm{~nm} / 10 \mathrm{~nm}$. (b) Average magnetic hysteresis loop of the sample with width/nominal thickness of $250 \mathrm{~nm} / 35 \mathrm{~nm}$. (c) Coercive field as a function of width for batch 1 ( $\left.t_{\text {Nom }}=25 \mathrm{~nm}\right)$ with positive and negative applied magnetic field (blue squares and red circles respectively). Please, be aware that the red circles partially hide the blue squares. The line is a fit to Equation 1. (d) Coercive field as a function of $t_{\text {Nom }}$ for batch 2 (width $=250 \mathrm{~nm}$ ) with positive and negative applied magnetic field (blue squares and red circles respectively). The line is a visual guide. 
perform micromagnetic simulations to investigate this possibility. Given the previous knowledge regarding the influence of the halo [31] and the effective magnetic shape [27] in the coercive field of FEBID magnetic nanostructures, it is convenient to know these properties in the studied nanowires. This is why we first carried out transmission electron microscopy (TEM) experiments to investigate the exact shape and composition of the nanowires, so that the subsequent micromagnetic calculations could reliably reproduce the observed dependence of the coercive field.

\section{TEM experiments: deposit shape, halo exten- sion, surface oxidation}

TEM characterization of the microstructure and composition of Fe nanowires was carried out in a probe-corrected FEI Titan 60-300 operated at $300 \mathrm{kV}$. Two TEM lamellae of Fe nanowires with a width of $250 \mathrm{~nm}$ and nominal thicknesses of 10 and $45 \mathrm{~nm}$ were fabricated using a focused $\mathrm{Ga}^{+}$ion beam and standard lift-out procedures in an FEI Helios 600 Nanolab. The slices were cut perpendicular to the nanowire length to analyze their cross-sectional width profile. The morphology and microstructure were determined by bright field (BF) TEM and high resolution TEM (HRTEM) imaging, and chemical composition of the sections was determined by combining high angle annular dark field (HAADF) imaging and electron energy loss spectroscopy (EELS) in scanning transmission electron microscopy (STEM) mode. The beam current in the STEMEELS experiments was $250 \mathrm{pA}$. Focused beam induced annealing effects were not observed in the experiments. These are easily observable because the images change with time, which was not the case in the experiments presented here.

Low-magnification BF-TEM images of the cross-sections of $\mathrm{Fe}$ nanowires are shown in Figure $3 \mathrm{a}$ and Figure $3 \mathrm{~b}$. These wires have been chosen for the TEM study because they are respectively the thinnest and the thickest ones for the fixed width of $250 \mathrm{~nm}$. Both wires presented irregular bell-shaped profiles, which are fitted using the following empirical formula:

$$
z=A \tanh \left[C\left(\left|y-y_{\mathrm{c}}\right|-y_{0}\right)\right]+z_{0},
$$

where $A$ and $C$ are fitting parameters while $y_{\mathrm{c}}$ corresponds to the peak position in the lateral $y$ direction (in this case manu-

(a)

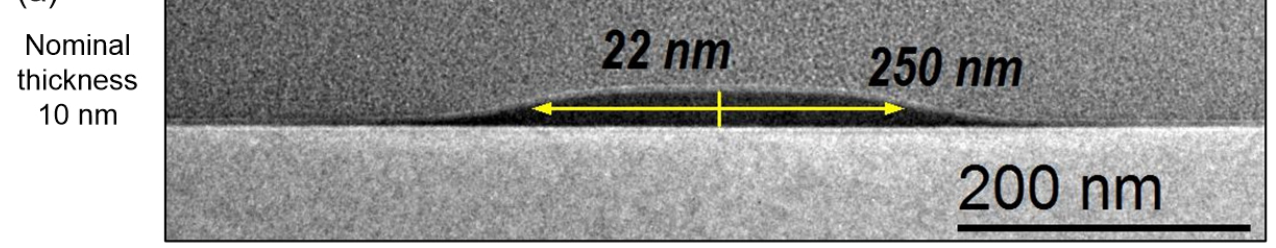

(b)

Nominal thickness $45 \mathrm{~nm}$
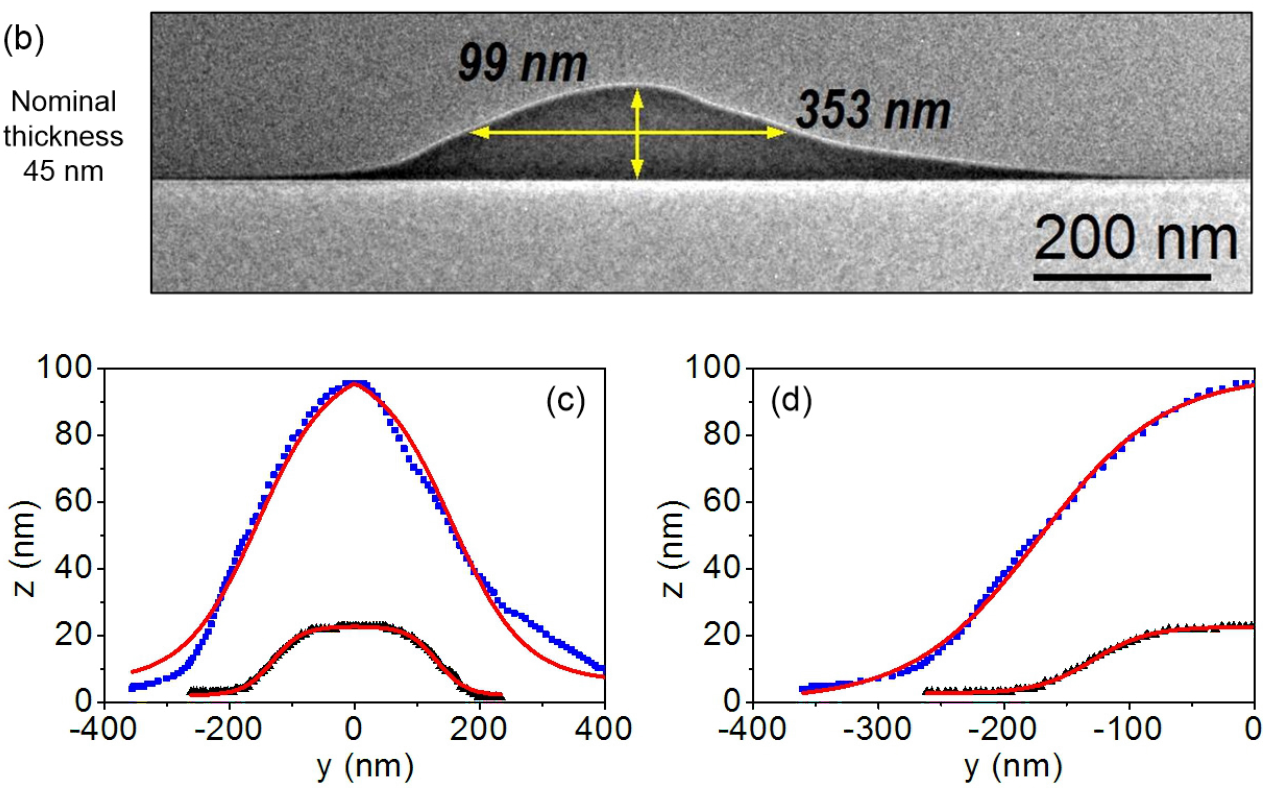

Figure 3: Low-magnification TEM images of the iron nanowires with width of $250 \mathrm{~nm}$ and nominal thickness of (a) 10 and (b) $45 \mathrm{~nm}$. Fitting of the (c) full and (d) half profiles of both nanowires using Equation 2. 
ally centered, $y_{\mathrm{c}}=0$ ) and $2 y_{0}$ corresponds to the full width of the profile measured along the $y$ direction at a height of $z_{0}$. Mathematically speaking, $z_{0}$ will correspond to the height where the bell profile changes its concavity (inflexion point). As one can notice in Figure 3c and Figure 3d, Equation 2 fits well the profiles of the nanowires extracted from TEM images of Figure 3a and Figure 3b. A better fit is obtained if we only take into account a half part of the profiles. All fitted parameters of both cases are reported in Table S3 (Supporting Information File 2). Comparing the values obtained from the fit for $2 y_{0}$ and $z_{0}$, one can note that both correlate, respectively, with the nominal values of the width and thickness of these two nanowires, being more similar for the thinnest one. In addition, the TEM images clearly reveal that the bell-shaped profile of the nanowires makes that the $t_{\mathrm{Nom}}$ is almost half of the maximum thickness $\left(t_{\mathrm{Max}}\right)$, corresponding to the peak height. Taking this fact into account and given that we have experimentally measured the profiles of the thinnest and the thickest nanowires, a linear extrapolation of the fitting parameters of Equation 2 permits to estimate the profile parameters of the intermediate thicknesses. The values of this extrapolation are reported in Table S2 (Supporting Information File 2), considering the fitted parameters obtained from Figure $3 \mathrm{~d}$ (half profile) and establishing $z_{0}$ as the $t_{\text {Nom }}$ of the nanowires. The HRTEM images of the nanowires, shown in Figure S3 (Supporting Information File 1), indicate that the $\mathrm{Fe}$ is nanocrystalline, as previously reported $[14,18]$. This microstructure will produce negligible magnetocrystalline anisotropy effects and, as a consequence, shape anisotropy will determine the magnetic anisotropy of the wires. The Fe content determined by EELS inside the wires is around $85 \%$, in good agreement with the EDS performed inside the FIB-SEM equipment. According to previous studies, the saturation magnetization in Fe deposits grown by FEBID corresponds well with the Fe content $[14,41]$. Then, one would expect a saturation magnetization about $80-85 \%$ of that of bulk Fe.

From the STEM-EELS experiments shown in Figure 4, where the oxygen intensity is probed from above the surface towards the interior part of the nanowires, it can be concluded that the top surface of the nanowires exhibits an oxidized layer of 4-5 nm thickness. The stoichiometry of this oxidized layer is found to be $\mathrm{Fe} / \mathrm{O}=1: 1(49.4 \pm 2 \%$ of $\mathrm{Fe}$ and $50.6 \pm 2 \%$ of $\mathrm{O})$, which corresponds to a paramagnetic material at room temperature. Such an oxidized surface is not ferromagnetic, which affects the overall magnetization reversal of the wires. The oxidized layer will have a strong impact on the thinner part of the wires, i.e., the tails and halo, which will be prone to lose the ferromagnetism. As a consequence, the effective ferromagnetic volume of the wire will be more localized towards the centre of the nanowire, thus modifying its functional ferromagnetic shape.


Figure 4: Compositional analysis through EELS of the iron nanowires with nominal thickness of (a) $10 \mathrm{~nm}$ and (d) $45 \mathrm{~nm}$. In (b) and (e) a close-up of the corresponding halos is shown. In (c) and (f) the intensity profiles of the oxygen from above the surface towards the interior part of the two nanowires (at the positions marked with yellow arrows) are shown: The thickness of the oxidized surface layer (FeO) is around 4-5 nm. 


\section{Micromagnetic simulations}

Quasi-static micromagnetic simulations were carried out to study the influence of the shape profile of the Fe nanowires and oxidized surface layer on the magnetization reversal process. We have focused our attention on the dependence of $H_{\mathrm{C}}$ on the thickness, which deviates from the model of magnetization reversal for nanowires described by Equation 1. In particular, we have studied the variation of $H_{\mathrm{C}}$ with the thickness for $250 \mathrm{~nm}$ wide Fe nanowires considering the actual geometry of the Fe nanowires of batch 2. The GPMagnet software package [42] was used to perform the simulations employing the following magnetic parameters for polycrystalline pure iron [43]: saturation magnetization $=1.7 \times 10^{6} \mathrm{~A} / \mathrm{m}$, exchange constant $=2.1 \times 10^{-11} \mathrm{~J} / \mathrm{m}$, anisotropy constant $=0$ (we assume that in nanocrystalline iron the magnetocrystalline anisotropy is averaged out). For the simulation, we have adopted the same in-plane geometry used for the MOKE measurements shown before. In order to decrease the simulation time, different cell sizes have been used depending on the thickness (see Table S1 in Supporting Information File 2). $H_{\mathrm{C}}$ has been calculated for three different cases, which allows us to investigate in a more general way the influence of the shape of the nanowires in the behavior of the coercive field:

case I: a rectangular profile where constant width $(250 \mathrm{~nm})$ is considered, case II: the actual bell-shaped profile determined from the TEM measurements, and case III: a reduced bellshaped profile considering a surface oxidation layer of $5 \mathrm{~nm}$ along the whole deposit.
The three different cases are schematically shown in Figure 5 for the Fe nanowire with a $t_{\mathrm{Nom}}$ of $20 \mathrm{~nm}$. As one can notice in Figure 5, in case I a single value of thickness can be considered, corresponding to the maximum height of the rectangular profile (i.e., $t=t_{\text {Nom }}=t_{\text {Max }}$ ). However, in the nanowires of cases II and III we defined $t_{\text {Nom }}=z_{0}$ and $t_{\text {Max }}=z_{\text {Max }}$ (peak height). The three-dimensional (3D) shape of the nanowires was designed by stacking several layers of equal thickness and either using the same area in the $x, y$ plane (for case I) or progressively reducing their widths in order to approach the bell-shape of the profile (for case II and III). The sketch shown in Figure 5c provides a visual understanding of the $3 \mathrm{D}$ structure of the simulated $\mathrm{Fe}$ nanowires. To simulate the oxidized bell-shaped profile, the structural bell-shaped profile has been reduced $5 \mathrm{~nm}$ from the surface in order to keep only the magnetic volume. It should be pointed out that temperature is not taken into account in the simulations. This is why the absolute value of $H_{\mathrm{C}}$ in the simulations is expected to be twice or more the experimental value at room temperature [27].

Plots of the simulated $H_{\mathrm{C}}$ values as a function of the nominal thickness for the three cases considered are presented in Figure 6. For case I (rectangular profile), in the range of thickness $5 \mathrm{~nm}<t_{\text {Nom }}<30 \mathrm{~nm}$, one notices that $H_{\mathrm{C}}$ increases with the thickness, as expected from Equation 1, until reaching a critical thickness $\left(t_{\mathrm{c}}\right)$ of $30 \mathrm{~nm}$. At that point, $H_{\mathrm{C}}$ exhibits a maximum. Above $t_{\mathrm{c}}, H_{\mathrm{C}}$ decreases with thickness. A similar behavior is observed in the nanowires with bell-shaped profiles, with and without the oxidized top layer of $5 \mathrm{~nm}$ (cases II and
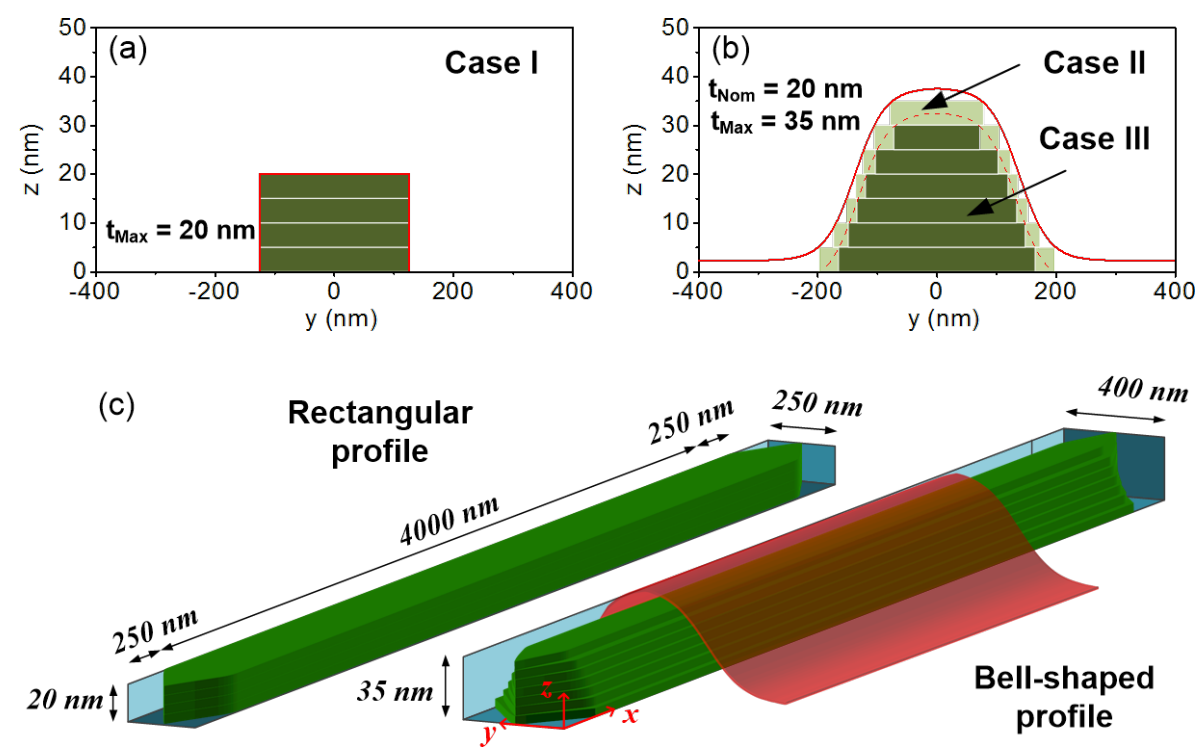

Figure 5: Sketch of the two-dimensional ( $y, z$ plane) geometrical shapes used in the micromagnetic simulation for a $250 \mathrm{~nm}$ wide Fe nanowire with a $t_{\text {Nom }}$ of $20 \mathrm{~nm}$ : (a) Rectangular profile (case I), (b) approximated bell-shaped profiles without (case II) and with (case III) an oxidized surface layer of $5 \mathrm{~nm}$. (c) Three-dimensional representation of the same wire in case I (left) and case II and case III (right). 
III, respectively). However, one can notice that the bell-shaped profile favors the occurrence of the maximum value of $H_{\mathrm{C}}$ at a lower $t_{\mathrm{Nom}}\left(t_{\mathrm{c}}=15 \mathrm{~nm}\right.$, for both cases II and III), also reducing the values of $H_{\mathrm{C}}$ for the same $t_{\mathrm{Nom}}$ compared to case I. Above $t_{\mathrm{C}}$, we note that the decreasing of $H_{\mathrm{C}}$ with the thickness observed in the nanowires of cases II and III resembles the experimental result (shown in Figure 2d). The full behavior of the simulated $H_{\mathrm{C}}$ as a function of the thickness in the bellshaped $\mathrm{Fe}$ nanowires resembles the one that is reported for $500 \mathrm{~nm}$ wide L-shaped Co-FEBID nanowires [40]. In [40], a maximum value of $H_{\mathrm{C}}$ is obtained at the crossover between two types of DWs nucleated in the corner of the "L". The transition is from transversal DWs (at low thicknesses) to vortex DWs (at high thicknesses). In principle, a change in the magnetization

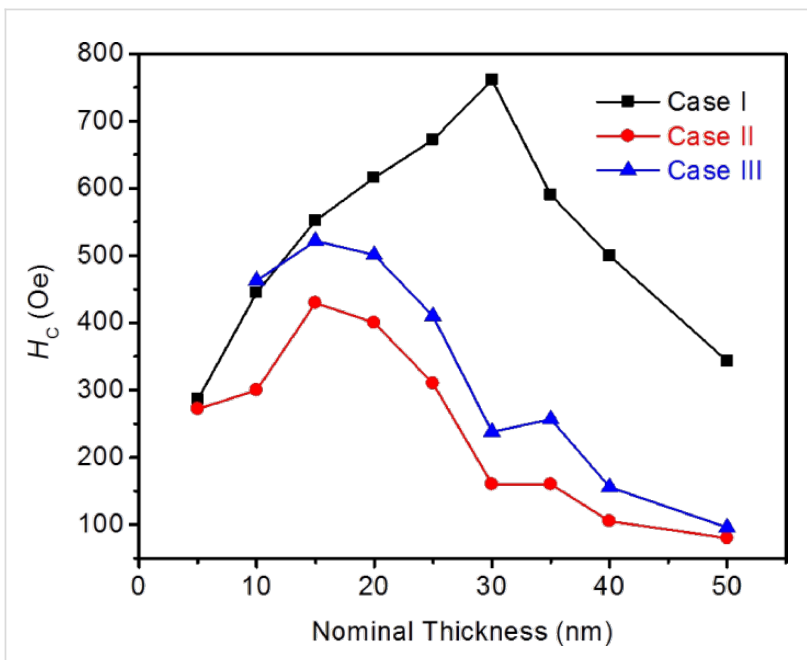

Figure 6: Coercive field $\left(H_{\mathrm{C}}\right)$ obtained from the simulations of iron nanowires with nominal width of $250 \mathrm{~nm}$ and varying nominal thickness (from 5 to $50 \mathrm{~nm}$ ) for the three cases discussed in the text. reversal mode is a good candidate to produce a dependence of $H_{\mathrm{C}}$ with thickness presenting a maximum. The micromagnetic simulations in the Fe nanowires permit a direct visualization of the magnetic configuration during magnetization reversal, which allows us to explore the changes in the reversal magnetization mechanism between nanowires with low and high thickness.

In Fe nanowires with rectangular profile (case I), for values of thickness $t_{\mathrm{Nom}}<t_{\mathrm{c}}\left(t_{\mathrm{c}}=30 \mathrm{~nm}\right)$, we find that the reversal magnetization process occurs through the propagation of two extended domain walls (EDWs). These relatively complex head-to-head and tail-to-tail EDWs are first nucleated at the ends of the nanowire and then propagate along the nanowire until they meet at the nanowire center and annihilate. Figure 7 shows five snapshots of the magnetization reversal process in the sample with width of $250 \mathrm{~nm}$, thickness of $20 \mathrm{~nm}$ and profile defined as case I. This magnetization reversal mechanism is not via the formation of a multi-domain structure but monodomain-type, with EDW nucleation at the pointed ends of the nanowires and propagation towards the center.

Interestingly, the simulations performed give access to image the $x, y$ plane-view magnetization states along the full depth of the nanowires. One example is the top and bottom layers of the $20 \mathrm{~nm}$ thick nanowire with rectangular profile, shown in Figure $8 \mathrm{a}$. The EDWs observed in this nanowire consist of small (extending only locally) $180^{\circ}$ and $90^{\circ} \mathrm{DWs}$, where the local magnetization rotates coherently in the plane of the nanowire ( $x, y$ plane) without out-of-plane magnetization rotations. This is in agreement with the observed magnetization reversal through coherent rotation modes that occurs at low thickness of magnetic nanowires $[39,44]$. This behavior can also

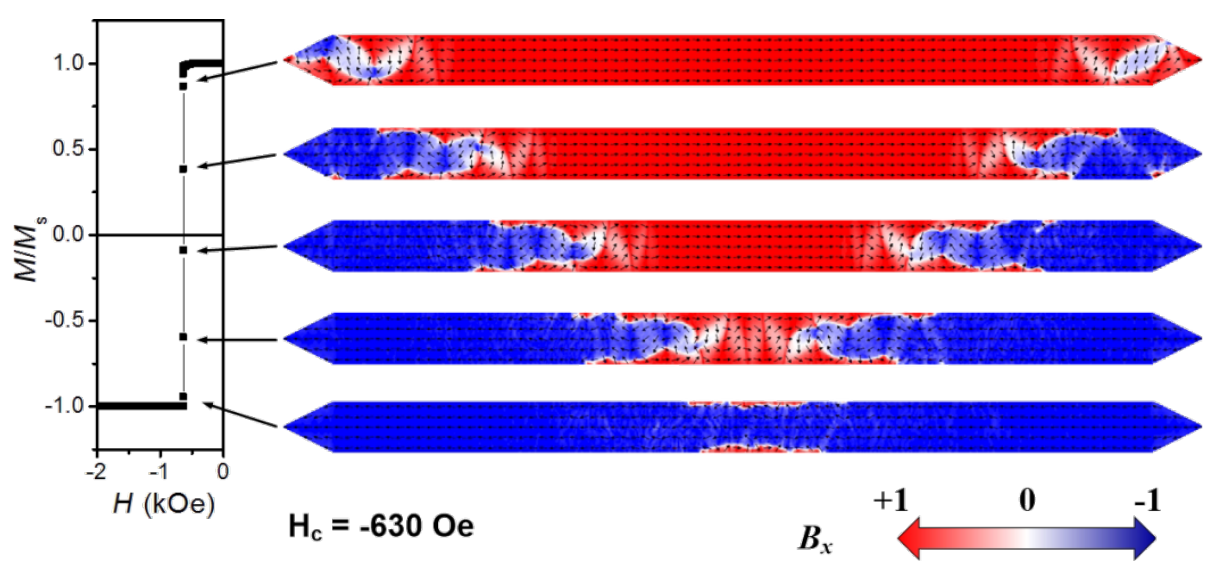

Figure 7: Simulated magnetization reversal with five snapshots of the magnetization state in the nanowire with width of $250 \mathrm{~nm}$, thickness of $20 \mathrm{~nm}$ and profile defined as case $\mathrm{I}$. 
be observed in cross-sectional images of the magnetization extracted along an EDW ( $y, z$ plane), where the local magnetization rotation across the whole thickness of the DWs occurs in the $x, y$ plane (see the $y, z$ plane view included in Figure 8a). Thus, the magnetic structure of the EDW is composed by small DWs covering the whole thickness and propagating to produce the magnetization reversal. This magnetization reversal mechanism, corresponding to a coherent rotation mode, is theoretically well described by Equation 1 [37]. Therefore, an increasing value of $H_{\mathrm{C}}$ with the thickness is expected in that nanowire thickness range. For $t_{\mathrm{c}}<t_{\text {Nom }}<50 \mathrm{~nm}$ simulations also indicate a monodomain-type reversal magnetization, through nucleation and propagation of EDWs, even though their small constituent DWs present local non-coherent rotation modes. This effect can be noticed in the cross-sectional images of the magnetization extracted across the EDWs for the $35 \mathrm{~nm}$ thick Fe nanowire of rectangular profile depicted in Figure $8 \mathrm{~b}$. Both, the pseudo-vortex wall formed in the inner part of EDW structure and the extended transversal $180^{\circ} \mathrm{DW}$ formed in the external part of the EDW structure, reveal local out-of-plane magnetization rotations of either Neél-type or C-shape magnetization distributions. In thicker films (above $t_{\mathrm{c}}$ ) the formation of DWs with non-coherent magnetization rotation is energetically more favorable than having coherent magnetization rotation $[44,45]$. The crossover to non-coherent magnetization reversal modes will be accompanied by a decrease of the DWs energy with the thickness. A direct consequence of this fact is that $H_{\mathrm{C}}$ will decrease with the thickness, as experimentally observed in Figure 2d.

The same reversal magnetization mechanisms previously observed in the rectangular profile nanowires have been found in the bell-shaped profile nanowires (case II and III). Thus, for $t_{\mathrm{Nom}}<t_{\mathrm{c}}$, with $t_{\mathrm{c}}=15 \mathrm{~nm}$, a monodomain-type reversal magnetization of EDWs with coherent rotation occurs, as shown in Figure 9a for a bell-shaped profile nanowire (case II) with $t_{\mathrm{Nom}}=10 \mathrm{~nm}$. For $t_{\mathrm{c}}<t_{\mathrm{Nom}} \leq 25 \mathrm{~nm}$, a monodomain-type reversal magnetization of EDWs with non-coherent rotation
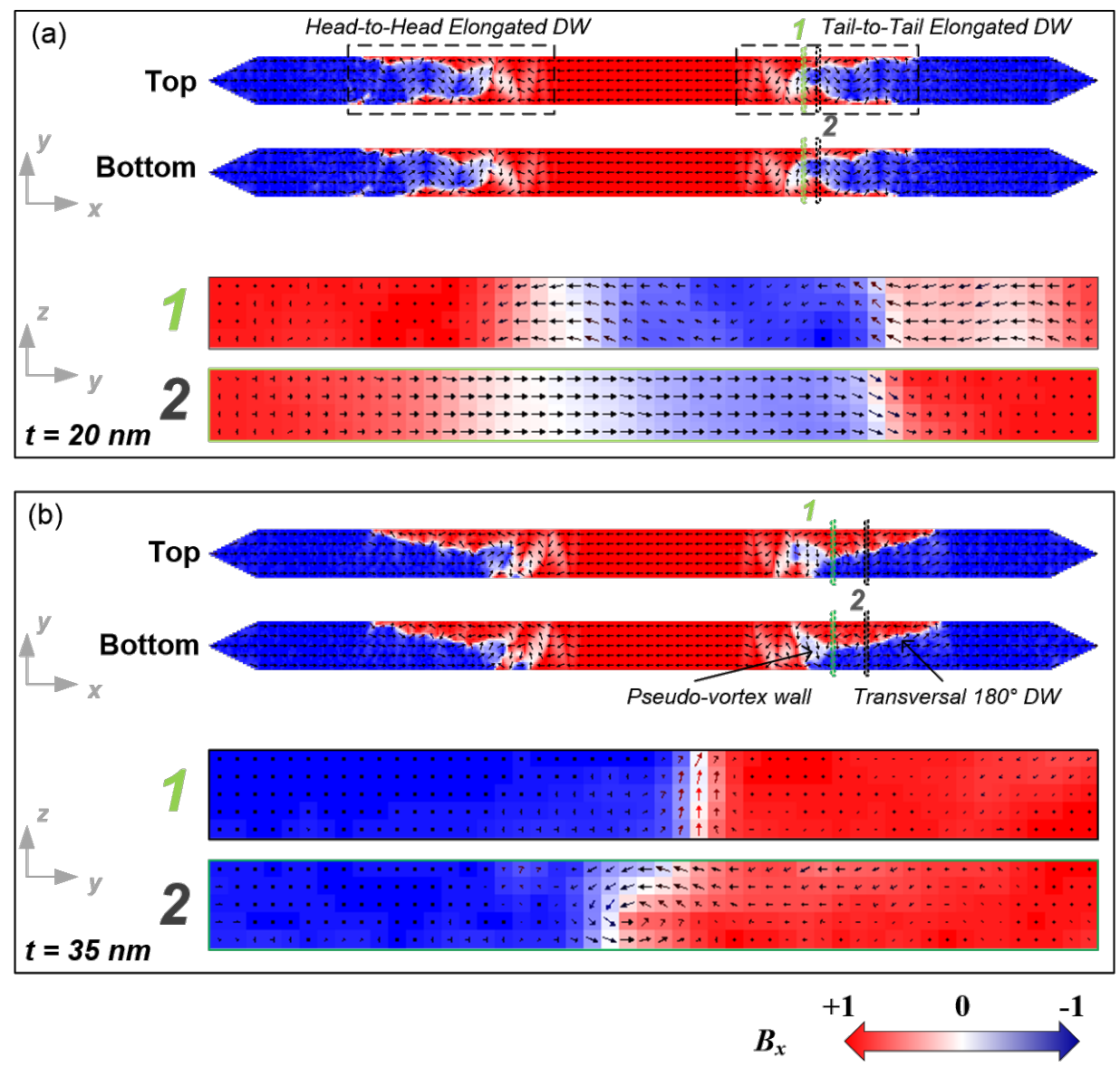

Figure 8: Magnetization vector-color maps extracted from the simulations for $250 \mathrm{~nm}$ wide Fe nanowires with rectangular profile (case I) and thickness of (a) $20 \mathrm{~nm}$ and (b) $35 \mathrm{~nm}$. The areas named "1" (green lines) and "2" (black lines) in the $x, y$ plane representations have been chosen for the representation in the $y, z$ plane. 

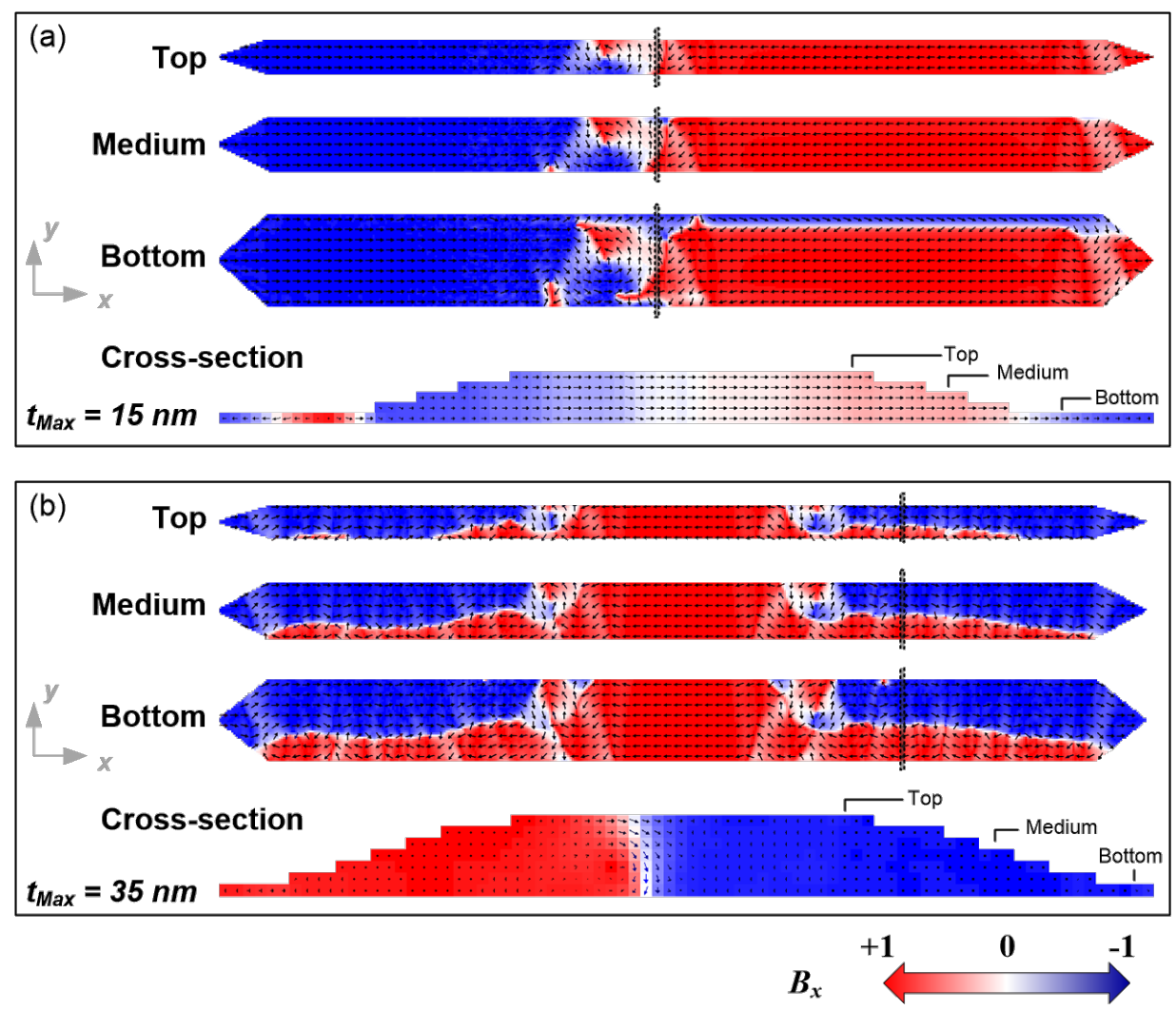

Figure 9: Magnetization vector-color maps extracted from the simulations for $250 \mathrm{~nm}$ wide Fe nanowires with bell-shaped profile (case II) and nominal thickness of (a) $10 \mathrm{~nm}$ and (b) $20 \mathrm{~nm}$. The areas marked with black lines in the $x, y$ plane representations have been chosen for the representation in the $y, z$ plane (cross-section).

occurs, as shown in Figure $9 \mathrm{~b}$ for a bell-shaped profile nanowire (case II) with $t_{\mathrm{Nom}}=20 \mathrm{~nm}$.

Interestingly, for $30 \mathrm{~nm} \leq t_{\mathrm{Nom}} \leq 50 \mathrm{~nm}$, the reversal magnetization process is already multidomain-type, as shown in Figure 10. Indeed, for nominal thicknesses of 30 and $35 \mathrm{~nm}$, the switching is produced through the formation and displacement of several magnetic domains along the nanowire length. The example of the $30 \mathrm{~nm}$ nanowire is shown in Figure 10a. For $t_{\text {Nom }}=40$ and $50 \mathrm{~nm}$, the magnetization reversal is given by the displacement of two transversal $180^{\circ} \mathrm{DWs}$, each of them initiated at one of the pointed ends of the nanowires. In this displacement, while one extreme of the transversal $180^{\circ} \mathrm{DW}$ is pinned at the apex of the nanowire, the other extreme moves towards the center of the nanowire. When both DW meet at the center, they form a single DW spanning along the full length of the nanowire, forming a Landau-Liftshitz domain pattern, as displayed in Figure 10b.

An interesting behavior is observed if we plot the values of $H_{\mathrm{C}}$ as a function of $t_{\mathrm{Max}}$. This new representation, shown in Figure 11, indicates that the different reversal magnetization mechanisms are directly linked to the maximum thickness, independently of the shape of the profiles. The shape of the profile, however, allows for the tuning of the value of $H_{\mathrm{C}}$, being higher in the rectangular profile nanowires. The surface oxidation in the bell-shaped profile (giving rise to a reduction of the magnetic volume) produces an increase in the values of $H_{\mathrm{C}}$. In Figure 11, two additional values of $H_{\mathrm{C}}$ for rectangular profile nanowires with $t_{\mathrm{Max}}=t_{\mathrm{Nom}}=70$ and $90 \mathrm{~nm}$ are reported. The simulations show a non-coherent multidomain magnetization reversal in such nanowires, similar to that observed in the bellshaped profile nanowires in the same $t_{\text {Max }}$ range.

From the information obtained in the micromagnetic simulations, one can safely infer that the dependence of $H_{\mathrm{C}}$ on the thickness in Fe nanowires is directly related to the specific magnetization reversal mode taking place at that thickness range. While reversal modes via coherent magnetization rotation produce an increasing value of $H_{\mathrm{C}}$ with the thickness, reversal modes via non-coherent magnetization rotation make $H_{\mathrm{C}}$ decrease with the thickness. Extrapolating this analysis to the experimental results obtained, we conclude that the decreasing value of $H_{\mathrm{C}}$ with thickness observed in Figure $2 \mathrm{~d}$ is 

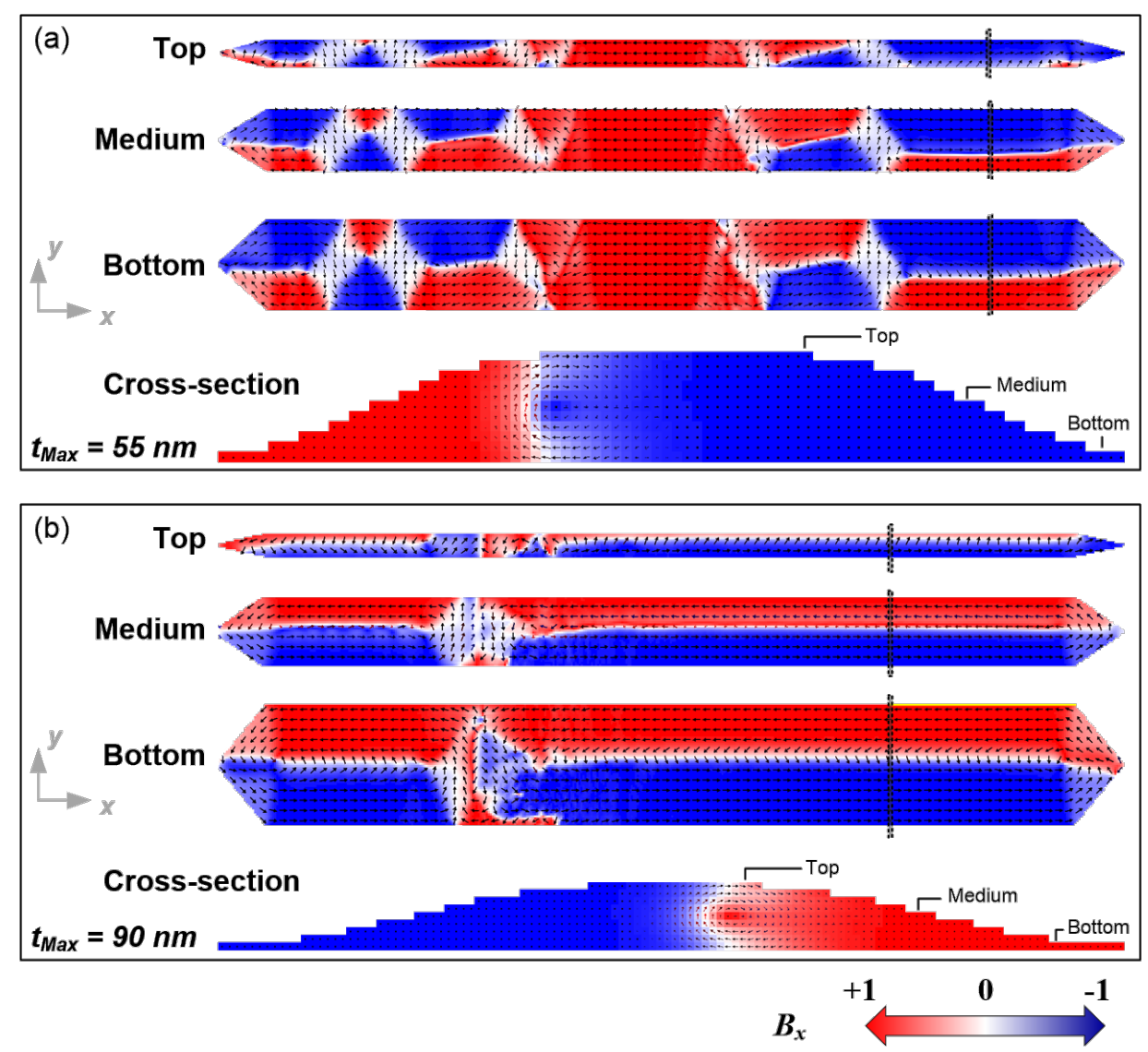

Figure 10: Magnetization vector-color maps extracted from the simulations for $250 \mathrm{~nm}$ wide Fe nanowires with bell-shaped profile (case II) and nominal thicknesses of (a) $30 \mathrm{~nm}$ and (b) $50 \mathrm{~nm}$. The areas marked with black lines in the $x, y$ plane representations have been chosen for the representation in the $y, z$ plane (cross-section).

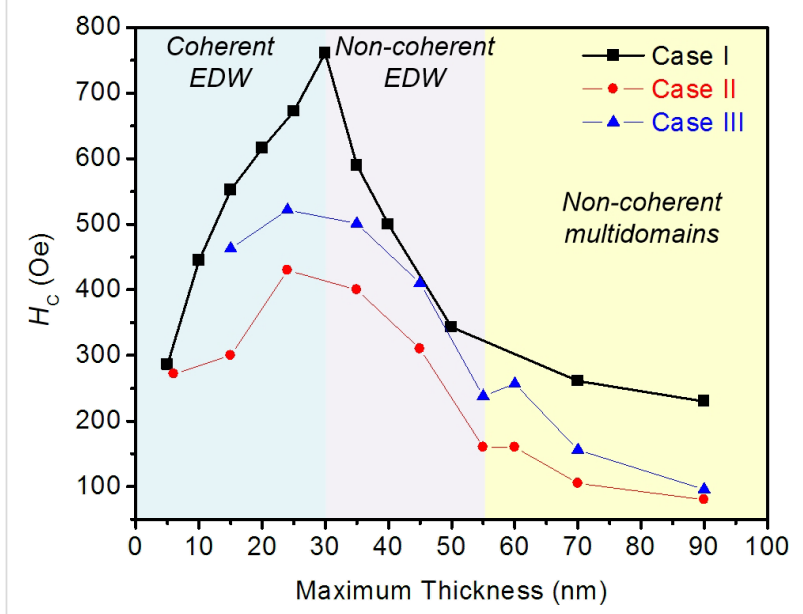

Figure 11: Coercive field $\left(H_{\mathrm{C}}\right)$ obtained from the simulations of nanowires as a function of $t_{\mathrm{Max}}$ for the three cases. caused by magnetization reversal modes with non-coherent magnetization rotation.

Having established the reason for the dependence of $H_{\mathrm{C}}$ on the thickness over the thickness range studied, a number of interesting features need to be discussed.

i) Effects produced by the bell shape and the halo of the nanowires. As shown in Figure 6, the bell-shaped nanowires present a lower $H_{\mathrm{C}}$ compared to the ones with a rectangular profile. This was already found in the simulations of FEBID Co nanowires performed by Fernández-Pacheco et al. [27]. The reason is that the nucleation of DWs is favored at the thinnest parts of the nanowire, which facilitates the magnetization reversal. Previous simulations in Fe nanowires considering an extended halo also highlighted its influence on the magnetization reversal [31]. Moreover, experiments where the halo was eliminated by means of ion irradiation already indicated its relevant role played in the magnetization reversal $[28,29]$. 
ii) Effects produced by the oxidized top layer of $5 \mathbf{~ n m}$. The oxidized top layer of $5 \mathrm{~nm}$ is expected to play some role in thin nanowires due to the modification of the magnetic properties of a substantial part of the nanowire. Assuming that this oxidized layer is paramagnetic at room temperature (as the Fe/O 1:1 stoichiometry suggests), this surface layer will lose its ferromagnetic behavior. According to the simulations shown in Figure 6 , the main effect of such oxidized layer is therefore to increase $H_{C}$. This is explained by the change in the ferromagnetic shape of the nanowire caused by the oxidation, which degrades or annihilates the ferromagnetism in the halo. The halo is a source for easy DW nucleation and its oxidation will weaken such mechanism for the initiation of the magnetization reversal.

iii) Behavior of $\boldsymbol{H}_{\mathbf{C}}$ at low thickness. The simulations predict that for the thinnest bell-shaped nanowires $\left(t_{\mathrm{Nom}}<15 \mathrm{~nm}\right)$, a regime of increasing $H_{\mathrm{C}}$ with thickness could be observed. This thickness regime was not experimentally addressed by us in a systematic way as it was beyond the scope of the present work. In fact, only one sample in our study falls in that thickness range, which does not permit to extract any reliable conclusion. However, it seems an interesting focus for future work. Such challenging work should consider, for example, a systematic characterization of the iron oxides formed at the surface, the continuity of the magnetic layer at such low thicknesses, and the nanowire roughness.

\section{Conclusion}

A systematic magneto-optical Kerr effect study of the coercive field as a function of thickness and width in $\mathrm{Fe}$ nanowires grown by focused electron beam induced deposition (FEBID) has been carried out. It has been found that the coercive field decreases for increasing thickness and width in the range of dimensions studied. In the particular case of $H_{\mathrm{C}}$ vs thickness for nanowires with constant width $(250 \mathrm{~nm})$, micromagnetic simulations have demonstrated that the decrease of $H_{\mathrm{C}}$ with thickness is due to the prevalent magnetization reversal mechanism, namely non-coherent magnetization reversal. In addition, micromagnetic simulations also show that the actual bell shape of the FEBID nanowires is important for the exact value of $H_{\mathrm{C}}$. The formation of a $5 \mathrm{~nm}$ surface oxidation layer on top of the nanowires has been observed experimentally. Micromagnetic simulations show that such surface oxidation produces a slight increase in $H_{\mathrm{C}}$. The results shown in this work demonstrate that $H_{\mathrm{C}}$ can be tailored in Fe nanowires grown by FEBID with appropriate control over their dimensions and shape, which is a crucial step towards the fabrication of functional devices based on these deposits such as magnetic memories, logic circuits and magnetic sensors. The reported micromagnetic simulations provide a detailed understanding of the magnetization reversal mechanisms in these $\mathrm{Fe}$ nanowires.

\section{Supporting Information}

\section{Supporting Information File 1}

Structural and compositional characterization of the iron nanowires.

[http://www.beilstein-journals.org/bjnano/content/ supplementary/2190-4286-6-136-S1.pdf]

\section{Supporting Information File 2}

Additional information about the profile shape of the nanowires and micromagnetic simulations.

[http://www.beilstein-journals.org/bjnano/content/ supplementary/2190-4286-6-136-S2.pdf]

\section{Acknowledgements}

This work was supported by Spanish Ministry of Economy and Competitivity through project No. MAT2011-27553-C02, MAT2011-28532-C03-02 and MAT2014-51982-C2-1-R, including FEDER funds and by the Aragon Regional Government. Authors would like to acknowledge the use of Servicio General de Apoyo a la Investigación-SAI, Universidad de Zaragoza.

\section{References}

1. De Teresa, J. M.; Fernández-Pacheco, A. Appl. Phys. A 2014, 117, 1645-1658. doi:10.1007/s00339-014-8617-7

2. Idigoras, O.; Nikulina, E.; Porro, J. M.; Vavassori, P.; Chuvilin, A.; Berger, A. Nanofabrication 2014, 1, 23-34. doi:10.2478/nanofab-2014-0003

3. Randolph, S. J.; Fowlkes, J. D.; Rack, P. D. Crit. Rev. Solid State Mater. Sci. 2006, 31, 55-89. doi:10.1080/10408430600930438

4. Utke, I.; Hoffmann, P.; Melngailis, J. J. Vac. Sci. Technol., B 2008, 26, 1197-1276. doi:10.1116/1.2955728

5. van Dorp, W. F.; Hagen, C. W. J. Appl. Phys. 2008, 104, 081301. doi:10.1063/1.2977587

6. Huth, M.; Porrati, F.; Schwalb, C.; Winhold, M.; Sachser, R.; Dukic, M.; Adams, J.; Fantner, G. Beilstein J. Nanotechnol. 2012, 3, 597-619. doi:10.3762/bjnano.3.70

7. Utke, I.; Hoffmann, P.; Berger, R.; Scandella, L. Appl. Phys. Lett. 2002, 80, 4792-4794. doi:10.1063/1.1489097

8. Boero, G.; Utke, I.; Bret, T.; Quack, N.; Todorova, M.; Mouaziz, S.; Kejik, P.; Brugger, J.; Popovic, R. S.; Hoffmann, P. Appl. Phys. Lett. 2005, 86, 042503. doi:10.1063/1.1856134

9. Fernández-Pacheco, A.; De Teresa, J. M.; Córdoba, R.; Ibarra, M. R. J. Phys. D: Appl. Phys. 2009, 42, 055005. doi:10.1088/0022-3727/42/5/055005

10. Belova, L. M.; Dahlberg, E. D.; Riazanova, A.; Mulders, J. J. L.; Christoferson, C.; Eckert, J. Nanotechnology 2011, 22, 145305. doi:10.1088/0957-4484/22/14/145305

11. Kunz, R. R.; Mayer, T. M. J. Vac. Sci. Technol., B 1988, 6, 1557-1564. doi:10.1116/1.584214

12. Takeguchi, M.; Shimojo, M.; Furuya, K. Nanotechnology 2005, 16 , 1321-1325. doi:10.1088/0957-4484/16/8/057 
13. Lukasczyk, T.; Schirmer, M.; Steinrück, H. P.; Marbach, H. Langmuir 2009, 25, 11930-11939. doi:10.1021/la901612u

14. Lavrijsen, R.; Córdoba, R.; Schoenaker, F. J.; Ellis, T. H.; Barcones-Campo, B.; Kohlhepp, T. J.; Swagten, H. J. M.; Koopmans, B.; De Teresa, J. M.; Magén, C.; Ibarra, M. R.; Trompenaars, P.; Mulders, J. J. L. Nanotechnology 2011, 22, 025302. doi:10.1088/0957-4484/22/2/025302

15. Serrano-Ramón, L.; Córdoba, R.; Rodríguez, L. A.; Magén, C.; Snoeck, E.; Gatel, C.; Serrano, I.; Ibarra, M. R.; De Teresa, J. M. ACS Nano 2011, 5, 7781-7787. doi:10.1021/nn201517r

16. Nikulina, E.; Idigoras, O.; Vavassori, P.; Chuvilin, A.; Berger, A. Appl. Phys. Lett. 2012, 100, 142401. doi:10.1063/1.3701153

17. Belova, L. M.; Hellwig, O.; Dobisz, E.; Dahlberg, E. D. Rev. Sci. Instrum. 2012, 83, 093711. doi:10.1063/1.4752225

18. Gavagnin, M.; Wanzenboeck, H. D.; Belić, D.; Bertagnolli, E. ACS Nano 2013, 7, 777-784. doi:10.1021/nn305079a

19. De Teresa, J. M.; Córdoba, R. ACS Nano 2014, 8, 3788-3795. doi:10.1021/nn500525k

20. Fernández-Pacheco, A.; Serrano-Ramón, L.; Michalik, J. M.; Ibarra, M. R.; De Teresa, J. M.; O’ Brien, L.; Petit, D.; Lee, J.; Cowburn, R. P. Sci. Rep. 2013, 3, 1492. doi:10.1038/srep01492

21. Franken, J. H.; van der Heijden, M. A. J.; Ellis, T. H.; Lavrijsen, R.; Daniels, C.; McGrouther, D.; Swagten, H. J. M.; Koopmans, B. Adv. Funct. Mater. 2014, 24, 3508-3514. doi:10.1002/adfm.201303540

22. Chia, H.-J.; Guo, F.; Belova, L. M.; McMichael, R. D. Appl. Phys. Lett. 2012, 101, 042408. doi:10.1063/1.4738789

23. Lavenant, H.; Naletov, V.; Klein, O.; de Loubens, G.; Casado, L.; De Teresa, J. M. Nanofabrication 2014, 1, 65-73. doi:10.2478/nanofab-2014-0006

24. Jaafar, M.; Serrano-Ramón, L.; Iglesias-Freire, O.; Fernández-Pacheco, A.; Ibarra, M. R.; De Teresa, J. M.; Asenjo, A Nanoscale Res. Lett. 2011, 6, 407. doi:10.1186/1556-276X-6-407

25. Córdoba, R.; Fernández-Pacheco, R.; Fernández-Pacheco, A.; Gloter, A.; Magén, C.; Stéphan, O.; Ibarra, M. R.; De Teresa, J. M. Nanoscale Res. Lett. 2011, 6, 592. doi:10.1186/1556-276X-6-592

26. Fernández-Pacheco, A.; De Teresa, J. M.; Córdoba, R.; Ibarra, M. R.; Petit, D.; Read, D. E.; O'Brien, L.; Lewis, E. R.; Zeng, H. T.; Cowburn, R. P. Appl. Phys. Lett. 2009, 94, 192509. doi:10.1063/1.3139068

27. Fernández-Pacheco, A.; De Teresa, J. M.; Szkudlarek, A.; Córdoba, R.; Ibarra, M. R.; Petit, D.; O'Brien, L.; Zeng, H. T.; Lewis, E. R.; Read, D. E.; Cowburn, R. P. Nanotechnology 2009, 20 , 475704. doi:10.1088/0957-4484/20/47/475704

28. Serrano-Ramón, L.; Fernández-Pacheco, A.; Córdoba, R.; Magén, C.; Rodríguez, L. A.; Petit, D.; Cowburn, R. P.; Ibarra, M. R.; De Teresa, J. M. Nanotechnology 2013, 24, 345703. doi:10.1088/0957-4484/24/34/345703

29. Nikulina, E.; Idigoras, O.; Porro, J. M.; Vavassori, P.; Chuvilin, A.; Berger, A. Appl. Phys. Lett. 2013, 103, 123112. doi:10.1063/1.4821034

30. Plank, H.; Smith, D. A.; Haber, T.; Rack, P.; Hofer, F. ACS Nano 2012 , 6, 286-294. doi:10.1021/nn204237h

31. Gavagnin, M.; Wanzenboeck, H. D.; Belic, D.; Shawraw, M. M.; Persson, A.; Gunnarsson, K.; Svedlindh, P.; Bertagnolli, E. Phys. Status Solidi A 2014, 211, 368-374. doi:10.1002/pssa.201330114

32. Cowburn, R. P. J. Phys. D: Appl. Phys. 2000, 33, R1-R16. doi:10.1088/0022-3727/33/1/201

33. Casey Uhlig, W.; Shi, J. Appl. Phys. Lett. 2004, 84, 759-761. doi:10.1063/1.1645332
34. Leven, B.; Dumpich, G. Phys. Rev. B 2005, 71, 064411. doi:10.1103/PhysRevB.71.064411

35. Dumpich, G.; Krome, T. P.; Hausmanns, B. J. Magn. Magn. Mater. 2002, 248, 241-247. doi:10.1016/S0304-8853(02)00347-5

36. Brands, M.; Leven, B.; Dumpich, G. J. Appl. Phys. 2005, 97, 114311. doi:10.1063/1.1921343

37. Yuan, S. W.; Bertram, H. N.; Smyth, J. F.; Schultz, S. IEEE Trans. Magn. 1992, 28, 3171-3173. doi:10.1109/20.179748

38. Koehler, T. R.; Fredkin, D. R. IEEE Trans. Magn. 1991, 27 , 4763-4765. doi:10.1109/20.278939

39. Goolaup, S.; Singh, N.; Adeyeye, A. O.; Ng, V.; Jalil, M. B. A. Eur. Phys. J. B 2005, 44, 259-264. doi:10.1140/epjb/e2005-00122-7

40. Rodríguez, L. A.; Magén, C.; Snoeck, E.; Serrano-Ramón, L.; Gatel, C.; Córdoba, R.; Martínez-Vecino, E.; Torres, L.; De Teresa, J. M.; Ibarra, M. R. Appl. Phys. Lett. 2013, 102, 022418. doi:10.1063/1.4776709

41. Córdoba, R.; Lavrijsen, R.; Fernández-Pacheco, A.; Ibarra, M. R.; Schoenaker, F. J.; Ellis, T. J.; Barcones-Campo, B.; Kohlhepp, T. J.; Swagten, H. J. M.; Koopmans, B.; Mulders, J. J. L.; De Teresa, J. M. J. Phys. D: Appl. Phys. 2012, 45, 035001. doi:10.1088/0022-3727/45/3/035001

42. Lopez-Diaz, L.; Aurelio, D.; Torres, L.; Martinez, E.; Hernandez-Lopez, M. A.; Gomez, J.; Alejos, O.; Carpentieri, M.; Finocchio, G.; Consolo, G. J. Phys. D: Appl. Phys. 2012, 45, 323001 doi:10.1088/0022-3727/45/32/323001

43. Donahue, M. J.; Porter, D. G. OOMMF User's Guide Version 1.0; 1999

44. Skomski, R.; Zeng, H.; Sellmyer, D. J. J. Magn. Magn. Mater. 2002, 249, 175-180. doi:10.1016/S0304-8853(02)00527-9

45. Huo, S.; Bishop, J. E. L.; Tucker, J. W. J. Appl. Phys. 1997, 81, 5239-5241. doi:10.1063/1.364906

\section{License and Terms}

This is an Open Access article under the terms of the Creative Commons Attribution License (http://creativecommons.org/licenses/by/2.0), which permits unrestricted use, distribution, and reproduction in any medium, provided the original work is properly cited.

The license is subject to the Beilstein Journal of Nanotechnology terms and conditions: (http://www.beilstein-journals.org/bjnano)

The definitive version of this article is the electronic one which can be found at: doi:10.3762/bjnano.6.136 